\title{
Link Sleeping and Wake-up Optimization for Energy Aware ISP Networks
}

\author{
Obinna Okonor, Ning Wang, Zhili Sun and Stylianos Georgoulas \\ Centre for Communication Systems Research \\ University of Surrey, Guildford, Surrey \\ GU2 7XH, United Kingdom
}

\begin{abstract}
Reducing energy consumption in the Telecom industry has become a major research challenge to the Internet community. Towards this end, numerous research works have been carried out to mitigate the growth of energy consumption through intelligent network control mechanisms. This paper proposes a novel approach to achieving energy efficiency in ISP backbone networks according to dynamic traffic conditions. The main objective is to enforce as many links as possible to go to sleep during the off-peak time, while in event of traffic volume increase, the minimum number of sleeping links should be required to wake up to handle this dynamicity and in a way that this creates minimal or no traffic disruption. Based on our simulations with the GEANT and Abilene network topologies and their traffic traces respectively, up to $47 \%$ and $44 \%$ energy gains can be achieved without any obstruction to the network performance. Secondly, we show that the activation of a small number of sleeping links is still sufficient to cope with any traffic surge instead of reverting to the full topology or sacrificing energy savings as seen in some research proposals.
\end{abstract}

\section{INTRODUCTION}

The sharp increase in energy consumption of ISP networks across the Internet has stimulated significant research efforts in the community towards energy efficiency improvement. Till now, a number of strategies for achieving greener ISP networks have been identified, including the design of energy aware traffic engineering (ETE) mechanisms and new networking protocols that are able to save energy as an intrinsic function of the underlying network. In both cases, proposals have been made for reconfiguring network links to sleep mode whenever customer traffic is at its low level. Such sleeping adaptation can be either opportunistic or time driven.

In this paper, we propose two complementary algorithms for controlling link sleeping operations according to the dynamic traffic conditions. First of all, we aim to maximise the total number of sleeping links when the traffic volume is low, but without incurring traffic congestion due to overloading of the remaining links in the pruned/reduced topology. This is possible since most networks are usually over provisioned in terms of network link capacity. Therefore, our developed Link Sleep Optimization Algorithm (LiSOA) is employed to prune the full network topology according to the links' respective utilization rates. On the other hand, in the event of traffic upsurge, when the reduced topology is being enforced, we aim to identify the minimum number of sleeping links to wake up in order to handle this situation, while leaving as many sleeping links to continue in their standby mode. In addition, this wake up process is done in a manner that incurs as low traffic disruption as possible. This research is the first of its kind because we do not only maximize the number of sleeping links, but also propose a protective disruption-minimizing mechanism for the topology in case of traffic surge instead of sacrificing energy savings by restoring the full topology; we call the scheme Link Wake-up Optimisation Technique (LiWOT). This implies that we answered the key research question in energy-aware topology adaptation which is "what if traffic volume experiences an upsurge when links are sleeping?" Towards this end, we employ network monitoring as in [1] to realise LiWOT. In this approach, a control server monitors the network at a certain interval and detects any congested link - thanks to TE-LSA [2] as this does not need any modification of the traditional IP forwarding protocol. If there is/are any sleeping link(s) whose wake-up is able to divert traffic away from the congested link, the server is responsible to communicate with the head node of the sleeping link to wake it up. LiWOT as such generates one or multiple additional paths incurred by the wake-up of the originally sleeping links, such that excessive customer traffic can be diverted away from the congested links. Ultimately, the main objective of LiWOT scheme is to identify/minimise the number of sleeping links that are required to wake up to avoid traffic congestion due to upsurge. Without such a scheme, chances are that the network may suffer from continuous congestion, or the full network topology needs to be activated, in which case, energy efficiency will be sacrificed. Another novelty of our scheme is that we consider links that create no traffic disruption with higher priority to sleep or wake-up in order to reduce the degree of traffic instability caused by its diversion. Specifically, adding or removing these links incurs the diversion of the traffic only originated from the head node of that link, but not other traffics from remote sources. Invariably, the convergence period of the network (a key network performance evaluation factor) is no more an issue if only this class of links are added or removed from the network.

The rest of the paper is organised as follows: Section II discusses the related energy aware algorithms available in the literature. Section III presents the problem formulation while section IV describes all the attributes of link sleeping and link wake-up algorithms. In section $\mathrm{V}$, performance evaluation is analysed while section VI concludes the paper and gives directions for future work. 


\section{RELATED WORK}

Recent research has shown that the rate of energy consumption in the Internet is very high with the greater amount of consumed energy on idle period [14]. This can also be shown by the very low average link utilization of the networks in Table II, when using the full topology. For GEANT and Abilene, it is $5.83 \%$ and $4.66 \%$ respectively - a call for energy savings. Most current state of the art in network energy savings is geared towards pruning the full network topology and applying this topology on the network during the idle period using different algorithms. This is referred to as ETE mechanisms and is very promising since the number of pruned links equals the number of line cards which constitutes almost $43 \%$ of the total energy consumed by a router [3]. Router power consumption also does not scale with the current link load but the installed capacity $[4,5]$. That means that once a link is activated, it will consume a significant amount of energy, regardless of whether it is highly or lightly loaded. The reduced topology can now be used by applying the corresponding routing protocol. Another promising direction is on developing energy saving protocols or hardware (industry based). [6] is the first position paper to suggest many heuristic approaches for energy savings in the current Internet. They suggest: (a) putting to sleep some idle interfaces/nodes and network components, e.g. line card, crossbar, and main processor, and (b) route aggregation to few paths during idle/low activity periods. Towards these, many solutions have been developed and can be found in a survey of the state of art on energy efficient networks [7].

Based on sampling of different traffic matrices and on the assumption that most ISPs have regular traffic patterns [8], [9] explore the use of two different topologies. They propose an efficient approach with time-driven network topology configurations. According to the scheme, they prune the full topology by removing first, a number of least loaded links, augment the reduced topology with few additional links and identify the duration period for the application of this topology. This is different from our method because our pruning process is based on only one traffic matrix without any augmentation of links (link augmentation can be regarded as a backup scheme in case of traffic surge, therefore, saving less energy). Secondly, we do not assume that ISPs have regular traffic patterns since traffic flow is dynamic in nature. To limit the number of network configurations, [10] developed a model to compute the energy savings in an operational network. They conclude that two or three configurations can be enough to handle each traffic matrix. This does not guarantee network robustness to dynamic traffic conditions.

The authors of [1] propose an offline algorithm for computing a scheduled sleeping link group (SSLG) based on the physical network topology (T) and the currently valid traffic matrix. In their proposal, they employ IP Fast Reroute mechanism to divert traffic before links in the SSLG can simultaneously go to sleeping mode. [11] proposes a heuristic algorithm that is capable of switching off network nodes and links. They adopt the following policies in their algorithm random, least link, least flow and opt-edge search to evaluate the possibility of turning off some nodes and links under connectivity and QoS constraints. However, convergence issue which is a major concern in routing protocols remains a question in this paper. [12] proposes an Energy Aware Routing algorithm that is capable of powering off some network links during low traffic periods. They classify the routers as exporters (ER), importers (IR) and neutral routers. All routers compute the shortest path tree (SPT) using Dijkstra algorithm while IR's use the SPT of the selected ER to calculate own shortest path. [3] shows that energy efficient routing is NP-complete and also developed an algorithm to aggregate traffics/links for power savings. No proactive measure for traffic surge was considered.

\section{PROBLEM FORMULATION}

A directed and connected network graph $G$ consists of a set of vertices $V$ connected by a set of edges $E$ denoted as $G(V, E) . \quad V$ represents the network nodes/routers while $E$ represents the set of network links. $N=|V|$ is the total number of nodes and $L=|E|$ is the total number of links in the network. Each link is associated with a link weight for computing shortest paths in IP routing. We therefore formulate an integer linear programming of our problem where the objective function is to maximizing the energy saving gains $\left(P_{e f}\right)$ subject to flow conservation constraints and other constraints as explained below:

maximize: $P_{\text {ef }}$

subject to:

$$
\begin{aligned}
& \sum_{j=1}^{N} \ell_{i j}^{s d}-\sum_{j=1}^{N} \ell_{j i}^{s d}=\left\{\begin{array}{cl}
t^{s d}, & \forall s, d, i=s \\
-t^{s d}, & \forall s, d, i=d \\
0, & \forall s, d, i \neq s, d
\end{array}\right. \\
& \frac{1}{c_{i j}} \ell_{i j} \leq \phi \quad \forall\{i, j\} \in E \\
& \ell_{\text {sleep }}=\ell_{\text {stubs }} \cup \ell_{\text {transits }}
\end{aligned}
$$

where:

$t^{s d}=$ Total traffic demand from source to destination $(\mathrm{s}-\mathrm{d})$ $\ell_{i j}^{s d}=$ Traffic load on link $\ell_{i j}$ for the $\mathrm{s}-\mathrm{d}$ pair

$\ell_{i j}=$ Total traffic load on link $\ell_{i j}$

$\phi=$ Threshold value for network link utilization conditions.

$c_{i j}=$ Bandwidth capacity of any link $\ell(i \rightarrow j)$

$P_{e f}=\frac{\sum \mathrm{P}_{\text {full Topology }}-\left(\sum \mathrm{P}_{\text {prune Topology }}+\sum \mathrm{P}_{\text {wakeup links }}\right)}{\sum \mathrm{P}_{\text {full Topology }}}$

The main objective of our research work is to increase network energy saving gains as much as possible, which is stated in equation 1 . This is achieved by maximizing the number of removed links and minimizing the number of links that need to be woken up during congestion. Equation 2 is the traditional flow conservation constraint. In other to control the utilization of individual links, we set equation 3 . This equation controls the congestion level in the network and is the primary determinant for the number of links that can go to sleep or need to be woken up in the network. The higher the threshold value, the higher the chance of more links to put to sleep and fewer links to be woken up. Equation (4) ensures that all sleeping links constitute only the stub $\left(\ell_{\text {stubs }}\right)$ and transit $\left(\ell_{\text {transits }}\right)$ sleeping links and not active links. 


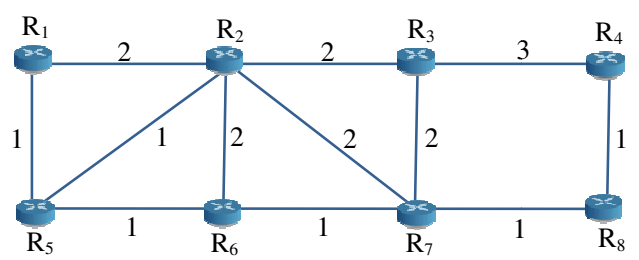

Fig.1. A synthetic network topology for illustrating the notion of stub links.

\section{SCHEME DESCRIPTION}

Using a simple synthetic network topology as shown in Fig. 1, we will discuss the basics of our algorithms. Before introducing our algorithm, we first classify network links into stub and transit links according to the physical topology and OSPF link weight setting as follows:

Stub link $\left(\ell_{\text {stub }}\right): \ell_{\text {stub }}$ is a network link solely used by its head node to route traffic in the network, and does not carry traffic originated from any other remote source nodes. It can be inferred that identifying stub links is based on the setting of OSPF link weights. From the synthetic network topology in Fig. 1 , we see that link $\ell\left(R_{2} \rightarrow R_{7}\right)$ can be classified as a stub link because it only carries traffic originated from its head node $R_{2}$. However, $\ell\left(R_{7} \rightarrow R_{2}\right)$ cannot be classified as such since some other upstream nodes can use it. Example, both $R_{7}$ and $R_{8}$ use $\ell\left(R_{7} \rightarrow R_{2}\right)$ to route traffic to $R_{2}$. However, both links of $R_{2}$ and $R_{6}$ are also stub links i.e. $\ell\left(R_{2} \leftrightarrow R_{6}\right)$.

Transit link $\left(\ell_{\text {transit }}\right)$ : In contrast, a transit link is allowed to carry traffic originated remotely. If a link is not a $\ell_{s t u b}$, then, it is referred to as a $\ell_{\text {transit. }}$. They are links that can be used by some upstream remote nodes to carry their traffic to downstream nodes. A good example of a $\ell_{\text {transit }}$ in Fig 1 is $\ell\left(R_{2} \rightarrow R_{3}\right)$ and $\ell\left(R_{7} \rightarrow R_{2}\right)$.

Sleeping link $\left(\ell_{\text {sleep }}\right)$ : These are links that are put to sleep mode from the physical topology in view of saving energy. Any sleeping link is either a sleeping stub link $\left(\ell_{\text {stubs }}\right)$ or a sleeping transit $\operatorname{link}\left(\ell_{\text {transits }}\right)$.

\section{A. Traffic Diversion}

We will analyze the difference between adding stub and transit links to the network topology. The identification of stub links is so as to prioritise them for sleeping reconfiguration in order to minimise routing instability. This is because the sleeping or waking-up of a stub link only requires local traffic diversion which can be directly handled by the head node of the stub link. None of the traffic originated from remote sources will get affected. It is also important to note that the number of stub links is solely dependent on the link weight settings and therefore can be manipulated to be higher than the number of transit links.

Let's consider two different scenarios on how these sleeping links behave as shown in Fig $2 \mathrm{a}$ and Fig $2 \mathrm{~b}$ respectively. In Fig $2 \mathrm{a}, \ell\left(R_{2} \rightarrow R_{7}\right)$ is a sleeping stub link. All links are symmetrical with maximum link capacity of $10 \mathrm{Mbps}$ and a network threshold value of $90 \%$. Assume that at time $\mathrm{t}_{1}$, the following source-destination pairs route $5 \mathrm{Mbps}$ of traffic in the network: $\left\{\mathrm{R}_{5}, \mathrm{R}_{7}\right\}$ through $R_{5} \rightarrow R_{6} \rightarrow R_{7}$, and $\left\{\mathrm{R}_{2}, \mathrm{R}_{7}\right\}$ through $R_{2} \rightarrow R_{6} \rightarrow R_{7}$.

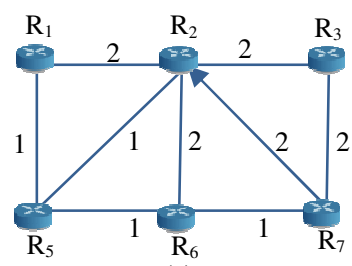

(a)

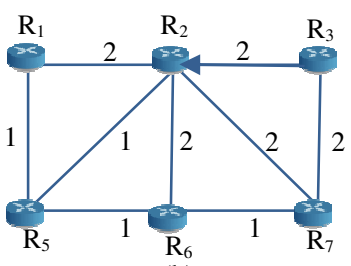

(b)
Fig.2. An illustration on how stub sleeping links behave in a network (a) and how transit sleeping links behave in a network (b)

Therefore, at $\mathrm{t}_{1}$, the link load of $\ell\left(R_{6} \rightarrow R_{7}\right)$ is $10 \mathrm{Mbps}$, implying a link utilization of $100 \%$ which is greater than the set threshold. If $\ell\left(R_{2} \rightarrow R_{7}\right)$ is added to the network, routing of $R_{5}$ does not change since it does not make use of that link. Only traffic from $R_{2}$ is diverted through $\ell\left(R_{2} \rightarrow R_{7}\right)$. However, in Fig 2b, the sleeping link is $\ell\left(R_{2} \rightarrow R_{3}\right)$ which is a transit link for $R_{5}$. Assume that at $\mathrm{t}_{1}, R_{2}$ and $R_{5}$ both send 5 Mbps of traffic to $R_{3}$ through $R_{2} \rightarrow R_{7} \rightarrow R_{3}$, and $R_{5} \rightarrow R_{6} \rightarrow$ $R_{7} \rightarrow R_{3}$ respectively. There will be congestion at $\ell\left(R_{7} \rightarrow\right.$ $\left.R_{3}\right)$ and if $\ell\left(R_{2} \rightarrow R_{3}\right)$ is added to the network to resolve such congestion, all traffics from $R_{2}$ and $R_{5}$ will be diverted through it. This, of course does not solve any congestion but rather diverts the congestion from $\ell\left(R_{7} \rightarrow R_{3}\right)$ to $\ell\left(R_{2} \rightarrow R_{3}\right)$. Therefore, before addition of any transit link, routing of remote traffics must be considered while stub links do not in any way affect any remote routings except for their traffics, which also reduces the traffic disruption incurred by remote traffic rerouting.

\section{B. Link Sleeping Optimization Algorithm (LiSOA)}

Most networks are over provisioned in terms of links and link capacities, which provides the opportunity of reconfiguring links to sleep especially when the traffic volume is low. Fig. 3 shows our proposed algorithm for calculating the sleeping links in the network.

The algorithm takes as input, the full network topology, a given traffic matrix, and a threshold value which is determined by the operator. First of all, the utilization of each network link is computed based on the mapping of a traffic matrix to the physical topology. In order to minimise routing instability caused by link removal, we first start the removal process from the stub links. This is because the removal of stub links only causes the diversion of the traffic originated from their head node, but no traffic originated remotely is affected. In this case, the list containing stub links is first arranged in ascending order of their link utilization rates. This arrangement order solves the issue of not prioritizing heavily loaded links in the removal process but rather, underutilized links which are easier to have their traffic diverted and go to sleep mode. Secondly, the diversion of underutilized link load in the network is much easier than that of a loaded link and also considering the high rate of underutilization in today's network.

Before any link is removed, a connectivity check is carried out. This is to confirm that the network is not a spanning forest. More so, upon consideration of a link removal, the link utilization in the reduced topology needs to be updated. If the maximum link utilization (MLU) of the network does not exceed a pre-defined threshold, the link can be successfully removed for sleeping. Otherwise, the next stub link is checked. 
Upon successful removal of any link, the network utilization is updated. In an event of any unsuccessful removal, the link is inserted back to the network. This continues until all stub links have been investigated. When this is done, the algorithm repeats the same process using as input, the current network utilization and the remaining links (i.e. active links that do not contain the removed stub links). This implies that those removed stub links will not be considered in the network utilization rate, and therefore are referred to as sleeping links. In the removal process of the remaining active links, the same process of stub removal is repeated for each active links. At the end, the remaining links in the network are referred to as the active topology which will be deployed to the network during off peak period while all the sleeping links are kept for wake up process by LiWOT if there is any congestion as a result of traffic surge. Therefore, the algorithm returns the active topology and the list of sleeping links whose line cards will be powered off for energy saving purposes.

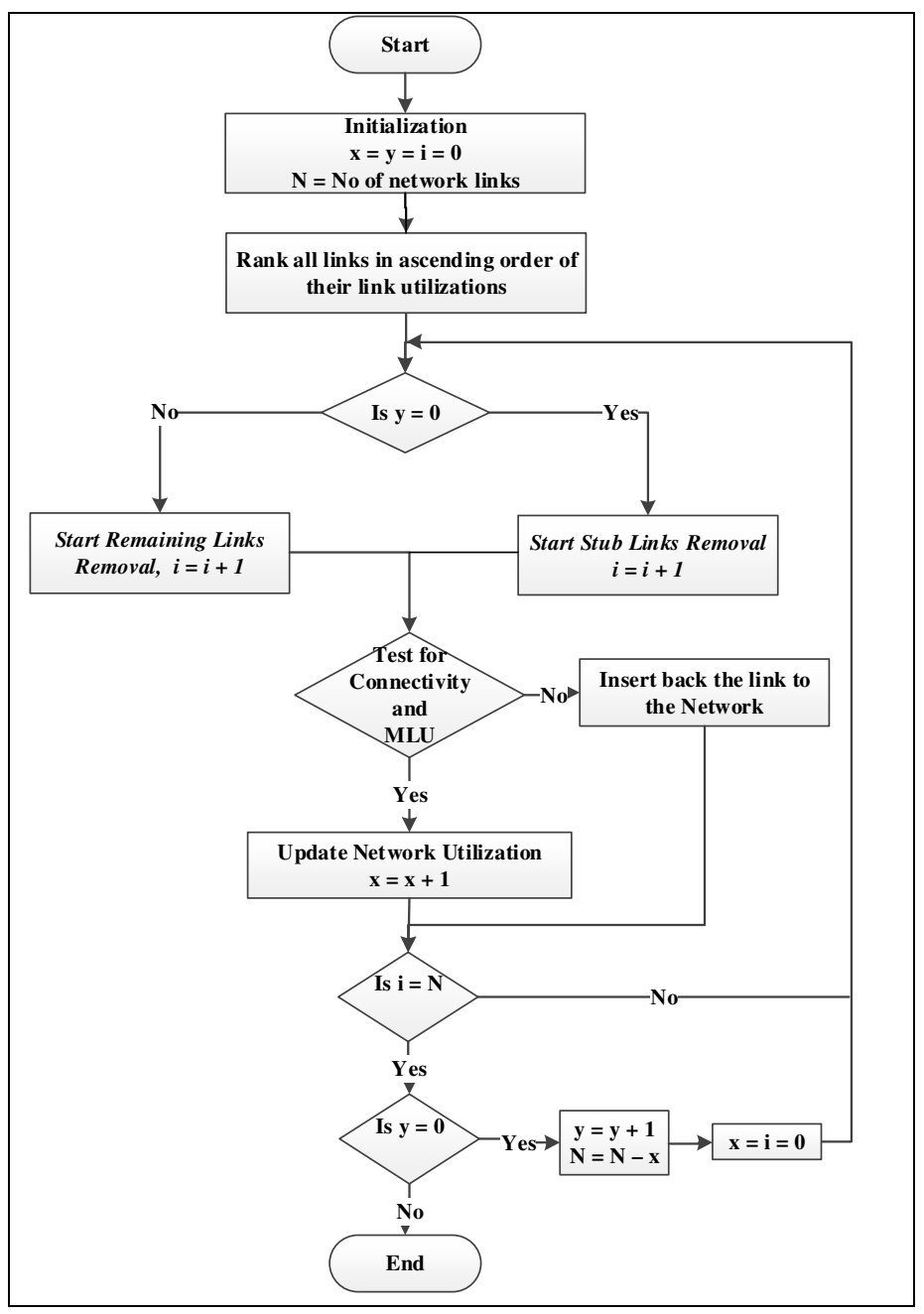

Fig. 3. Proposed heuristic algorithm for link removal.

\section{Link Wake-up Optimization Technique (LiWOT)}

LiWOT, as an online process according to traffic dynamicity, can be realised at a server which receives network monitoring on all links through TE-LSAs, and hence is able to quickly detect any congestion. At certain set periods of time, the server runs LiWOT to ascertain the utilization of the network. Once it exceeds the threshold value, it determines the minimum number of links to wake up in other to support the traffic surge. The algorithm first identifies the head node of the congested links and the traffic flow for possible diversion through alternate paths. These traffic flows across the link are sorted in a descending order with respect to their demands. The main idea of this descending order approach is to prevent waking up of many sleeping links, since the minimum number of flows (causing the link load to be above the utilization threshold) will need to be diverted instead of many low demand flows possibly scattered along different paths. This, in addition to maintaining energy saving gains, also further reduces the traffic instability due to traffic diversion.

This process is done for all set of sleeping links while prioritizing stub links. It implies that once a stub link resolves all network congestion, no other sleeping link is considered. In stub link consideration, only the source nodes of the traffic flow are identified. Each packet flow consists of source and destination address in its packets header. Therefore, LiWOT considers if the source node of each packet has any stub link that once activated will divert the traffic away from the congested link. For each of the traffic flows in the list, the algorithm checks for any possible sleeping link that can wake up and divert traffic away from the congested link. If such link exists, then it wakes up that link and the MLU is recalculated. If MLU is less than the set threshold, the current topology is returned; otherwise, the next entry in the list is checked. When all stub links have been checked and there is still congestion, the algorithm repeats the process with transit links.

In transit links consideration, due to how they route traffics, both the source and destination nodes of the traffic flow are considered for any alternative path. Once there is any sleeping link that can divert away traffic from the congested link, such link is inserted to the network. The MLU is also recalculated for network congestion status. If such addition resolves the network congestion, the algorithm returns the current topology (the current topology is the reduced topology plus added sleeping $\operatorname{link}(\mathrm{s}))$. It is also worth noting that this addition of links to the network minimizes routing disruption. This is a result of the removal process which must also conform to equation (4). If the search of links from stub and transit links does not resolve the current network congestion status, the last check is made on the full topology traffic path. Each link is checked on its merit and added as such. However, this last step is not exploited in our simulation but can be viewed as a backup scheme. The only two added links for both $90 \%$ and $95 \%$ threshold values happen at the second stage of the algorithm without requiring any further complementary action. It is worth noting that network update is performed explicitly in the logical network which the controller maintains through TELSA before applying it to the real network to avoid instability. 


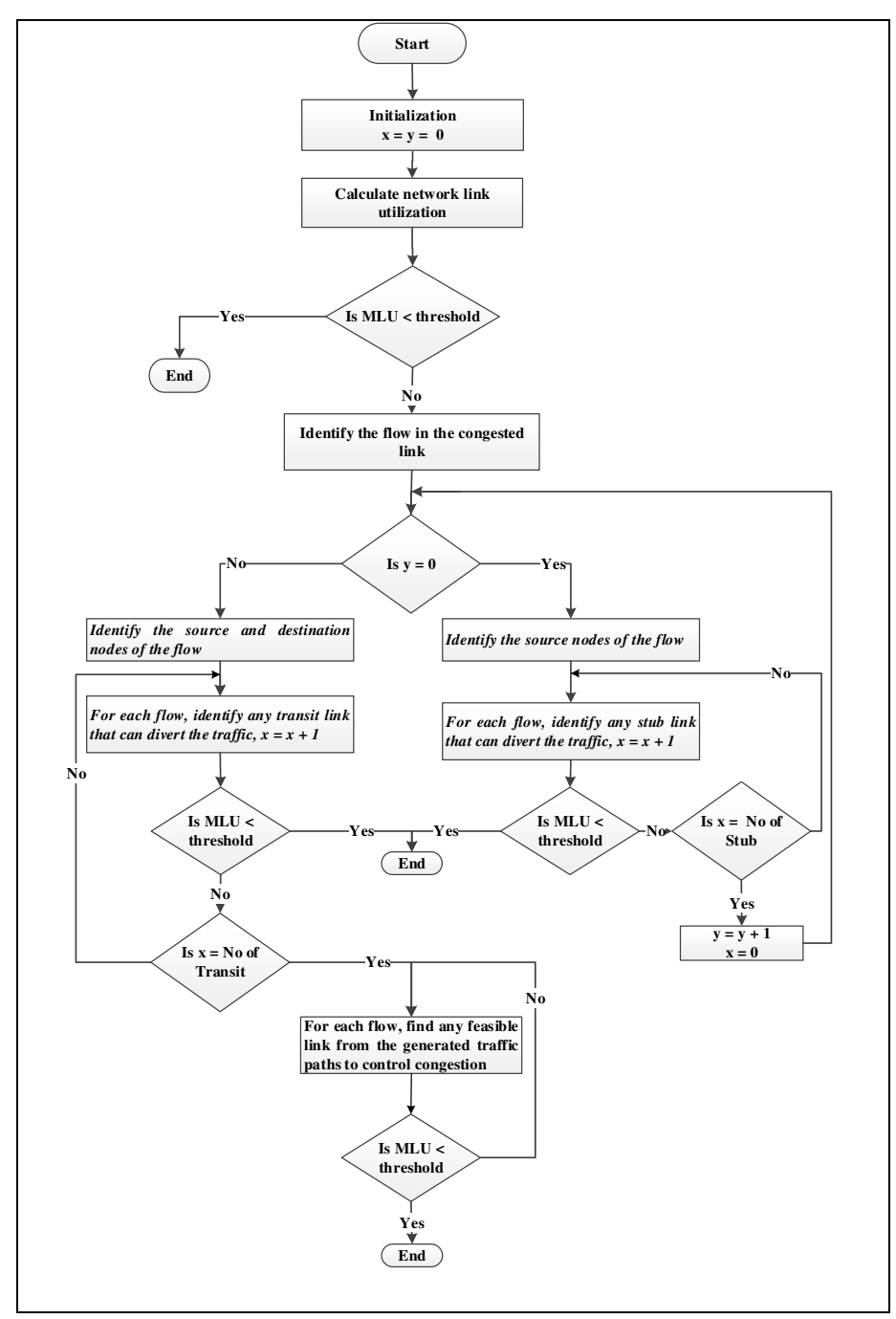

Fig. 4. Proposed LiWOT algorithm for link wake-up

TABLE I. POWER CONSUMPTION RATE OF ROUTER LINE CARDS [13]

\begin{tabular}{|c|c|c|}
\hline Line card & Speed (bps) & Power (Watts) \\
\hline 1-Port OC-192 & 9953280 & 174 \\
\hline 2-Port OC-48 & 4976640 & 160 \\
\hline 1-Port OC-48 & 2488320 & 140 \\
\hline 1-Port OC-3 & 155520 & 60 \\
\hline
\end{tabular}

\section{PERFORMANCE EVAlUATION}

This section presents the performance of our simulated results using the GEANT and Abilene network topologies and their published traffic traces. The GEANT network topology contains 23 Point-of-Presence (PoP) nodes, 74 unidirectional inter-PoP links of varying delay and bandwidth capacities while Abilene contains 12 PoP nodes, 30 unidirectional interPoP links of varying delay and bandwidth capacities.

\section{A. Performance Analysis of LiSOA}

Our algorithm, when applied to both network topologies, is able to maximize energy savings without incurring any congestion to the network. This shows energy savings of over $44 \%$ during the considered period if we take the energy consumption rate as shown in Table I. In our case, we use a threshold of $100 \%$ in the removal process and also to confirm with equation (3) - the higher the threshold value, the higher the number of links to be removed. On the choice of the traffic matrix (TM), we considered 7 days TMs and found out that the TM with the least MLU contains less utilized origindestination pairs on average, hence our choice of using it. However, some researchers consider many TMs in the period, starting with the TM with the least MLU, and then the TM with highest MLU. The simple reason is to augment the topology with more links in the removal process since there is no proactive congestion control mechanism, thereby wasting more energy. More so, we start our removal process from the least loaded link as seen in some literature. We also took into account that the actual energy consumption of these links is not based on their link utilization rates but on the power consumption of the line cards [4].

In other to minimize traffic disruption during the considered period and maximize energy savings, links were removed in ascending order of their MLU ratings prioritizing stub links. Our simulation is based on 7 days of traffic traces, and the utilization threshold for link removal is set to $100 \%$. Another important aspect of LiSOA is that it employs the use of a realistic TM in the removal process and therefore, enhances the robustness of its residual topology to dynamic traffic since that is expected to evolve around the realistic TM, even though the realistic TM considered represents the most optimistic case in terms of network traffic status. More so, our approach also guarantees higher link removal as seen in the pruning ration in Table II. After link removal, the topology is then referred to as the reduced topology which is applied to the network at a point in time and is subsequently modified by LiWOT (called current topology) whenever a need arises before being restored again, when the traffic is at its lowest again (i.e. the reduced topology is restored periodically). Also, the performance of LiSOA can be seen more clearly in the performance of Abilene network where no link was added even after the removal of $57 \%$ of the network links (see Fig. 6 where TMs were mapped to the full and pruned topologies). This is due to the removal procedure. However, for GEANT topology as seen in Fig 5, only addition of two links could guarantee such output (see Table II).

\section{B. Performance Analysis of LiWOT}

The main strength of LiWOT is that it does not consider traffic as having a regular pattern form. This implies that it can handle both regular patterned GEANT and irregular patterned Abilene TMs. At each interval, when LiWOT is run, it calculates the network link utilization to ascertain the current network state. Once there is congestion, the algorithm checks for a minimum number of links to correct it. This is achieved by prioritizing stub links in the process, then the transit links before checking the traffic paths from the original topology. At the particular time that LiWOT is applied, the considered congestion is network based and not link based. This implies that even if there are more than one congested links in the network, the algorithm detects and rectifies them all. In order to avoid congestion from occurring and to exploit LiWOT proactively, the threshold should be set below $100 \%$. This is to give room for proactive congestion avoidance. A snapshot of 
the performance can be viewed in Fig. 5 and Fig. 6 for GEANT and Abilene network respectively. Different TMs corresponding to traffic evolution in time were mapped on both reduced and full topologies. Results show that despite the number of removed links in the pruned topology, its performance does not differ much from the full topology. However, the performance of the pruned topology comes with a cost of only two links needing to be woken up in other to maintain the set threshold value within the period in consideration. This implies that the topology was over provisioned by more than $50 \%$. This is not the same for Abilene network in Fig. 6 because of its irregular traffic pattern. The most important thing is that even with a lower number of network links, we can still achieve a congestion free network following our approach. In the diagram, one can see how MLU drops and goes up again. This depicts differences in peak and off peak periods. During peak periods, the MLU is very high and gradually returns to low values at off peak periods. At this set threshold, the MLU performance always satisfies the original threshold constraint as shown in equation (3). Our analysis was conducted for a period of one week and we can see that as the MLU of the full topology gets higher (peak period), the MLU of the current topology is also affected. However, this improves the average link utilization (ALU) of the network. Table II shows that using the topology produced by LiWOT, the ALU is about 3 times higher than that of the full topology of GEANT and even more for Abilene. This is an indication of a more balanced use of network resources and reduction in link underutilization. This is illustrated in Fig. 7 and Fig. 8 for both topologies. It also shows the effect of LiWOT in the current topology.

In regards to convergence avoidance which is of paramount in today's network, Table II shows the ratio of stub links to transit links in the network. It also depicts the convergence saving ratio. However, we control convergence by allowing wake up links to remain as active links. This implies that once a link is added to the network, its operation continues in active capacity until the reduce topology is reversed to. The reversal process is optional (daily/weekly) depending on the operator. In our case, we applied it for a period of one week after which the pruned topology is reapplied.

Due to the high level of underutilization of network resources, Fig. 7 and Fig. 8 show the advancement on the network average link utilization as compared to the full topology. This is also as a result of the performance of LiWOT, otherwise, the high utilization rate will lead to congestion. On the threshold choice, we found out that the lower the threshold value, the more the number of added links. This was confirmed with our simulated results at $90 \%$ for GEANT and 70\% for Abilene respectively. It then shows how important a congestion control algorithm is needed in today's network. More so, the added links do not in any way cause bouncing effects or count to infinity to the network.

The attributes in Table II are based on the performances of full and pruned topologies. As shown, 'a' in a/b depicts number of attribute while ' $b$ ' is the total value of the attribute. For example, 21/74 means 21 stub links in the full topology that has a total number of 74 links. T represents topology.
TABLE II. PERFORMANCE ANALYSIS OF TM DIURNAL IN 7 DAYS

\begin{tabular}{|c|c|c|}
\hline ATTRIBUTES & GEANT & ABILENE \\
\hline Location & Europe & US \\
\hline Stub in full T & $21 / 74$ & $4 / 30$ \\
\hline Transit in full T & $53 / 74$ & $26 / 30$ \\
\hline Removed stub from full T & $11 / 21$ & $2 / 4$ \\
\hline Removed transit from full T & $28 / 53$ & $15 / 26$ \\
\hline No of stubs in pruned T & $12 / 39$ & $10 / 17$ \\
\hline No of transit in pruned T & $27 / 39$ & $7 / 17$ \\
\hline Added links through LiWOT & 2 & 0 \\
\hline ALU of full T & $5.83 \%$ & $4.66 \%$ \\
\hline ALU of pruned T & $16.08 \%$ & $23.23 \%$ \\
\hline Pruning ratio & $53 \%$ & $57 \%$ \\
\hline Percentage energy saving & $47 \%$ & $44 \%$ \\
\hline
\end{tabular}

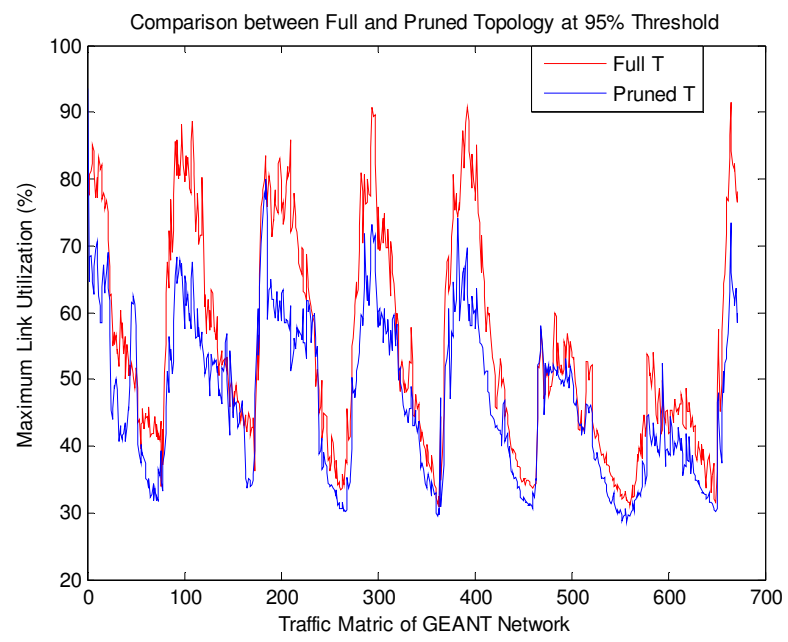

Fig. 5. Performance of LiWOT @ 95\% using GEANT diurnal TM.

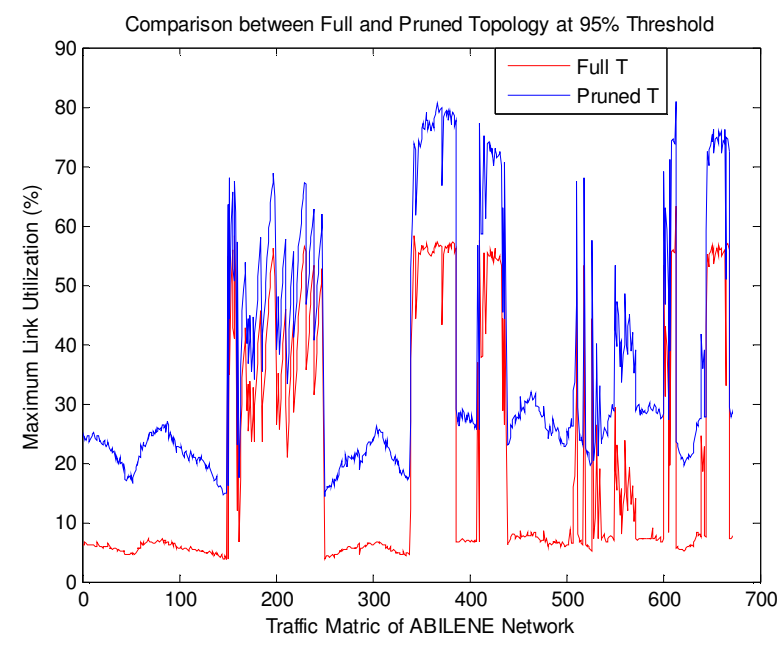

Fig. 6. Performance of LiWOT @ 95\% using Abilene diurnal TM. 


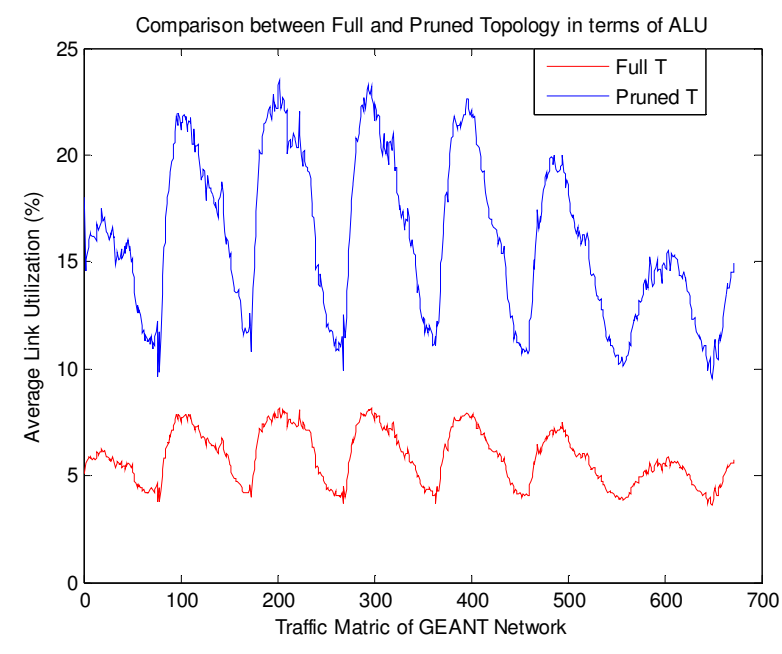

Fig. 7. ALU comparison between Full and Pruned topology of GEANT.

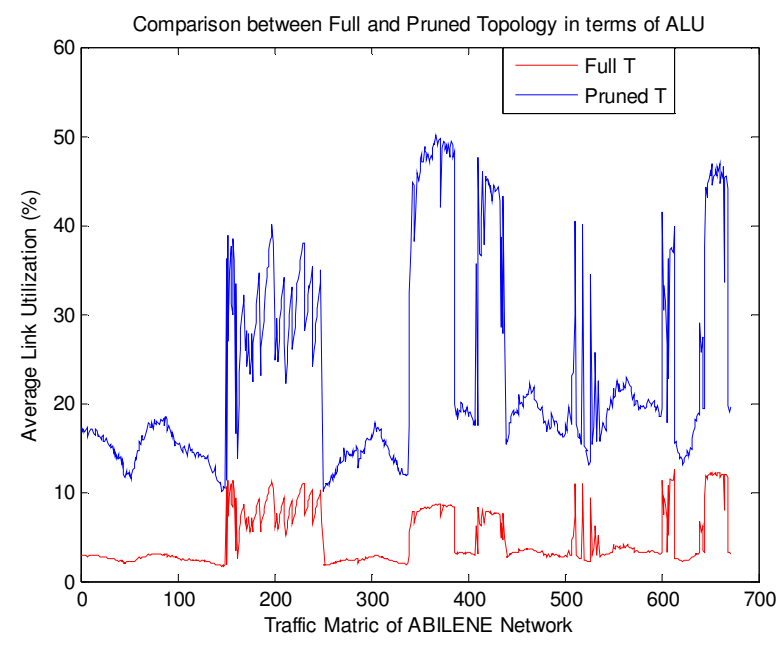

Fig. 8. ALU comparison between Full and Pruned topology of Abilene.

\section{CONCLUSION}

In this paper, we introduce two complementary link sleeping and wake-up algorithms for achieving energy efficiency under dynamic traffic conditions. The schemes are based on the prioritizing stub links for sleeping and subsequent wake up (when needed) in order to minimise routing instability. Our research shows a substantial improvement on energy savings of an operational ISP network compared to using the full network topology while keeping routing disruption at a minimum. Until now, most energy saving approaches has been geared towards sacrificing energy savings when there is any network traffic surge. Our approach gradually updates the network only when there is need for such update and in a way that minimizes routing disruption. The obtained results over realistic case studies show that our algorithm is able to actually adapt to traffic surges without causing any form of congestion when links are waken up. It is a novel approach to convergence avoidance in today's networks and this work piece has shown that adopting sleeping mode in network is a practical approach towards energy savings without incurring any congestion. More so, since stub links can be defined based on link weight settings, ISPs can actually enhance this process by increasing the number of stub links in their network. The impact of advertising and not advertising these links is also worth exploring. The way to address these research directions will be tackled in our subsequent work.

\section{ACKNOWLEDGMENT}

The first author will like to acknowledge the management of the Petroleum Technology Development Fund (PTDF) Nigeria for sponsoring his research work.

\section{REFERENCES}

[1] N. Wang, C. Michael, and K. H. Ho, "Disruption-Free Green Traffic Engineering with NotVia Fast Reroute" in IEEE Communications Letters, October 2011.

[2] D. Katz, K. Kompella, and D. Yeung, "Traffic Engineering (TE) Extensions to OSPF Version 2" IETF RFC 3630, September 2003.

[3] Q. Li, M. Xu, Y. Yang, L. Gao, et al, "Safe and Practical EnergyEfficient Detour Routing in IP Networks" in Networking, IEEE/ACM Transactions on (Volume:PP, Issue: 99) 2013.

[4] L. Chiaraviglio, M. Mellia, and F. Neri, "Energy-aware Backbone Networks: a case study" in International Conference on Communication (ICC), IEEE 2009.

[5] A. Adelin, P . Owezarski and T . Gayraud, "On the Impact of Monitoring Router Energy Consumption for Greening the Internet" in IEEE/ACM International Conference on Grid Computing (GRID), 2010.

[6] M. Gupta and S. Singh, "Greening of the Internet" in ACM SIGCOMM, Karlsruhe, Germany. August 2003.

[7] A. P. Bianzino, C. Chaudet, D. Rossi, and J Rougier, "A Survey of Green Networking Research" in IEEE Communication Surveys and Tutorials 14, 2012.

[8] M. Roughan, A. Greenberg, C. Kalmanek, M. Rumsewicz, J. Yates, and Y. Zhang, "Experience in measuring backbone traffic variability: models, metrics, measurements and meaning" in Proceedings of the 2nd ACM SIGCOMM Workshop on Internet measurement, ser. IMW 2002, New York, NY, USA: ACM, pp. 91-92.

[9] F. Francois, N. Wang, K. Moessner, and S. Georgoulas, "Optimization for Time-driven Link Sleeping Reconfigurations in ISP Backbone Networks" in Network Operations and Management Symposium (NOMS), IEEE, April 2012.

[10] L. Chiaraviglio and A. Cianfrani, "On the Effectiveness of Sleep Modes in Backbone Networks with Limited Configurations" in Software, Telecommunications and Computer Networks (SoftCOM), Italy, 2012.

[11] L. Chiaraviglio, M. Mellia, and F. Neri, "Reducing Power Consumption in Backbone Networks" in IEEE International Conference on Communications, Germany ICC 2009.

[12] A. Cianfrani, V. Eramo, M. Listanti, M. Marazza, and E. Vittorini, "An Energy Saving Routing Algorithm for a Green OSPF Protocol" in IEEE International Conference On Computer Communications (INFOCOM) 2010.

[13] Cisco 12000 series Packet Over SONET/SDH (POS) line cards "http://www.cisco.com/en/US/prod/collateral/routers/ps6342/product_da ta_sheet0900aecd803fd7b9.pdf"

[14] M. A. Rodrigues and K. G. Ramakrishnan, "Optimal Routing in Data Networks" International Telecommunication Symposium (ITS) 1994. 